\title{
Motility is required for the competitive fitness of entomopathogenic Photorhabdus luminescens during insect infection Catherine A Easom ${ }^{1,2}$ and David J Clarke*1
}

Address: ${ }^{1}$ Department of Microbiology, University College Cork, Ireland and ${ }^{2}$ Department of Biology and Biochemistry, University of Bath, BA2 7AY, UK

Email: Catherine A Easom - bs1cae@bath.ac.uk; David J Clarke* - david.clarke@ucc.ie

* Corresponding author

Published: 3 October 2008

BMC Microbiology 2008, 8:168 doi:10.1186/147I-2180-8-168

This article is available from: http://www.biomedcentral.com//47/-2/80/8//68

(c) 2008 Easom and Clarke; licensee BioMed Central Ltd

This is an Open Access article distributed under the terms of the Creative Commons Attribution License (http://creativecommons.org/licenses/by/2.0), which permits unrestricted use, distribution, and reproduction in any medium, provided the original work is properly cited.

\begin{abstract}
Background: Photorhabdus are motile members of the family Enterobactericeae that are pathogenic to insect larvae whilst also maintaining a mutualistic interaction with entomophagous nematodes of the family Heterorhabditiae. The interactions between Photorhabdus and its hosts are thought to be an obligate part of the bacteria's life-cycle in the environment. Motility often plays a key role in mediating bacteria-host interactions and, in this study, we were interested in characterising the role of motility in the Photorhabdus-nematode-insect tripartite association.
\end{abstract}

Results: We constructed deletion mutants of flgG (blocking flagella production) and mot $A B$ (blocking flagella rotation) in P. luminescens TTOI. Using these mutants we show that both the $\triangle$ flg $G$ and $\triangle$ motAB mutants are equally as good as the wild-type (WT) bacteria in killing insects and supporting nematode growth and development suggesting that flagella production and motility are not required for pathogenicity or mutualism. However we show that the production of flagella is associated with a significant metabolic cost during growth on agar plates suggesting that, although not required for pathogenicity or mutualism, there must be a strong selective pressure to retain flagella production (and motility) during the interactions between Photorhabdus and its different hosts. To this end we show that both the $\triangle$ flgG and $\triangle$ motAB mutants are out-competed by WT Photorhabdus during prolonged incubation in the insect revealing that motile bacteria do have a fitness advantage during colonisation of the insect larva.

Conclusion: This is the first report of a role for motility in Photorhabdus and we show that, although not required for either pathogenicity or mutualism, motility does contribute to the competitive fitness of Photorhabdus during infection of the insect (and, to a lesser extent, the nematode). This adaptive function is similar to the role ascribed to motility in mammalian pathogens such as uropathogenic Escherichia coli (UPEC). Therefore, in addition to describing a role for motility in Photorhabdus, this study reinforces the relevance and utility of this bacterium as a model for studying bacteria-host interactions.

\section{Background}

Photorhabdus are Gram negative bacteria that are highly virulent pathogens of a wide variety of insect larvae whilst also maintaining a mutualistic interaction with nematodes from the family Heterorhabditidieae. Photorhabdus normally colonise the intestinal tract of the infective stage 
of the nematode, the infective juvenile (IJ). The IJs are a non-feeding stage of the nematode that lives in the soil where they actively seek out potential larval hosts. On finding a suitable host the IJ enters the larva and the bacteria are regurgitated into the insect hemolymph. Photorhabdus actively circumvent the insect innate immune system by inhibiting and adapting to the humoral response whilst also suppressing phagocytosis by circulating haemocytes [1-3]. During infection of the insect the bacteria grow exponentially, producing a wide range of toxins and hydrolytic exoenzymes that are responsible for the death and subsequent bioconversion of the insect larva into a nutrient soup that is ideal for nematode growth and development $[4,5]$. The nematodes feed on the bacterial biomass within the insect cadaver and develop through juvenile (J1-J4) stages to form adult males and females. After several rounds of reproduction the J1 stage nematodes receive uncharacterised environmental cues that stimulate the development of IJs. At this point the developing IJs are colonised by Photorhabdus before they emerge from the insect cadaver to find new hosts (for recent reviews see [6,7]).

Many bacteria are motile through the action of large complex protein assemblages called flagella. The production and function of flagella are best studied in the enteric bacteria Escherichia coli and Salmonella enterica serovar Typhimurium where it has been shown that the expression of genes required for flagella-mediated motility (and chemotaxis) is controlled by a complex regulatory network $[8,9]$. Flagellum-mediated motility often plays a key role in mediating different bacteria-host interactions. For example motility is important during the colonisation of the squid by Vibrio fischeri and also during the infection of mammals by both Salmonella and E. coli [10-14]. Photorhabdus are motile through the action of peritrichously arranged flagella and we hypothesised that motility must play some role in the interactions between Photorhabdus and its invertebrate hosts. This was based on the principle that unused or redundant traits in bacteria will be lost over time [15]. Therefore we constructed specific flagellum-minus (Fla-) and non-motile (Mot-) mutants of Photorhabdus and, using these mutants, we show that motility is not required for either pathogenicity or mutualism. However we do show that WT bacteria out-compete nonmotile mutants during prolonged incubation in insect cadavers suggesting that motility confers a fitness advantage during colonisation of the insect.

\section{Results}

\section{Construction of mutations in flgG and motAB}

The genome sequence of $P$. luminescens TT01 is available and, by comparison with the closely related genome of $E$. coli, the TT01 genome is predicted to contain 49 genes required for the production and assembly of functional flagella and chemotaxis [16]. The motility-associated genes are found as 4 distinct genetic loci on a large (approx. $130 \mathrm{~Kb}$ ) fragment of TT01 genomic DNA stretching from nucleotide 2195317 to 2322562 (Fig. 1). To examine the role of motility in P. luminescens TT01 we constructed mutations in genes that are known to be required for motility in other bacteria, flgG and mot $A B$. The flgG gene encodes the distal rod protein of the flagellar hook-basal body (HBB) and mutations in flgG would be expected to prevent completion of this structure and, therefore, flagella assembly (Fla-). The mot $A B$ genes encode the motor-force generator that is required for rotation of the flagella and strains carrying mutations in $\operatorname{mot} A B$ can assemble normal flagella but the flagella cannot rotate (Mot-). Using a strategy that results in the construction of unmarked, non-polar deletions we completely removed the flgG gene such that only the start and stop codons remained. However the motAB operon overlaps with the downstream cheA gene and, to prevent any polar affects on cheA expression, the last 20 nucleotides of the motB gene (containing the predicted ribosome-binding site for the cheA gene) were not deleted. We confirmed that BMM800 $(\Delta f l g G)$ and BMM802 $(\Delta m o t A B)$ were non-motile using swim agar and that motility could be restored by the in trans expression of the respective genes from a plasmid i.e. pBMM800 (flgG) and pBMM802 (mot $A B$ ) respectively (Fig. 2).

\section{Motility is required for attachment to surfaces}

In $E$. coli (and other bacteria) it has been shown that flagella are important for bacterial attachment to abiotic surfaces [17-20]. Therefore the wells of a polypropylene microtitre plate were inoculated with TT01, BMM800 $(\Delta f l g G)$ and BMM802 ( $\triangle m o t A B)$ and the plate was incubated at $30^{\circ} \mathrm{C}$ without shaking for $24 \mathrm{~h}, 48 \mathrm{~h}$ and $72 \mathrm{~h}$. Bacterial attachment was quantified using crystal violet and it is clear that both the $\triangle f l g G$ and $\triangle$ mot $A B$ mutants were severely affected (5-10-fold) in their ability to attach to the walls of the microtitre plates when compared to the WT bacteria (Fig. 3). Attachment was restored to the mutants carrying a plasmid expressing the appropriate gene(s). Therefore, as in other enteric bacteria, motility is required for attachment of Photorhabdus to abiotic surfaces.

\section{There is a cost associated with the production of flagella}

Although flagella are generally not required for growth their production and assembly can be costly to the cell in terms of the utilisation of resources [21]. To determine whether the production of flagella is costly to Photorhab$d u s$ we set up a competition experiment whereby lipid agar plates were inoculated with 50:50 mixtures of WT bacteria with either the $\triangle f l g G$ or the $\triangle \operatorname{mot} A B$ mutant. The plates were incubated at $30^{\circ} \mathrm{C}$ and the relative abundance of each bacterial strain on the agar plate was measured at 

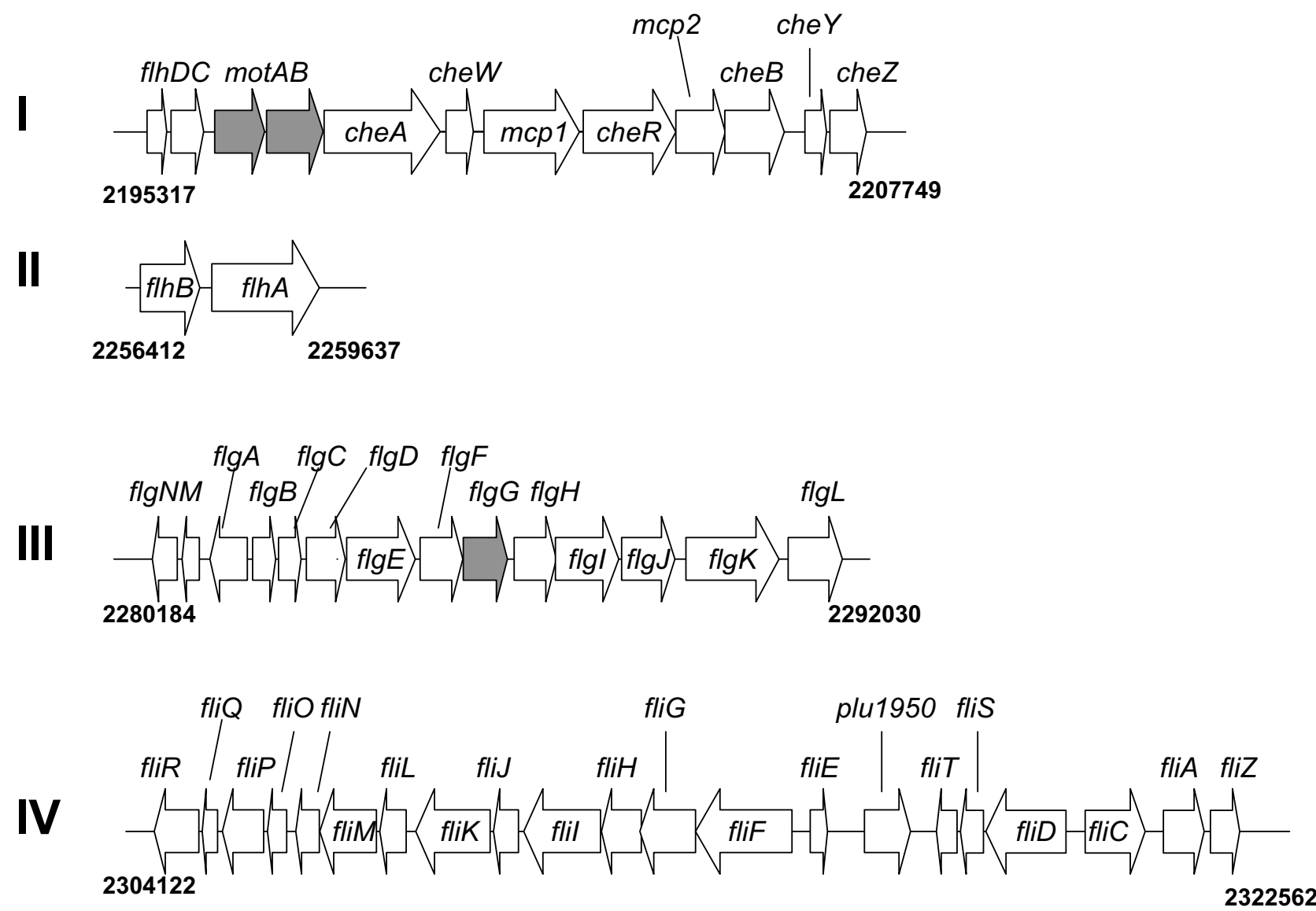

\section{Figure I}

The genetic loci encoding the genes required for flagella production and motility in $P$. luminescens TTOI. By comparison with the $E$. coli and Salmonella genomes (available at ColiBase http://colibase.bham.ac.uk) we identified 4 genetic loci (labelled I-IV) predicted to be involved in the production of flagella and chemotaxis in Photorhabdus. The numbers shown at beginning and end of each locus represents the genetic location on the Photorhabdus genome (according to PhotoList http:// genolist.pasteur.fr/PhotoList). The genes deleted in this study (motAB and $f l g G$ ) are indicated in grey.

time intervals post-inoculation i.e. 3 days and 21 days. In preliminary tests using LB broth we established that the growth rates of the $\triangle$ flgG and the $\triangle \operatorname{mot} A B$ mutants were identical to WT (data not shown). However, when grown for an extended period of time on lipid agar plates, we observed that the $\Delta$ flgG mutant was present at higher levels than the WT (a ratio of $85: 15$ on day 21) on the plate (Fig. 4A). On the other hand the $\triangle \operatorname{mot} A B$ mutant maintained a 50:50 ratio with the WT throughout the 21 days (Fig. 4B). Therefore there is a measurable cost associated with the production of flagella, but not motility per se, during Photorhabdus growth in vitro.

\section{Motility is required for the competitive fitness of Photorhabdus during insect colonization}

To test for a possible role during insect virulence, we injected $200 \mathrm{cfu}$ of TT01, BMM800 ( $\Delta \mathrm{flg} G)$ or BMM802 $(\triangle m o t A B)$ into final instar Galleria mellonella larvae. The $\mathrm{LT}_{50}$ (i.e. time taken to kill $50 \%$ of the insect larvae) values of the WT and mutant bacteria were similar showing that motility is not required for Photorhabdus virulence against insects (data not shown). To test whether motility might confer an advantage at some point during infection of the insect we injected a 50:50 mixture of TT01 with either the $\triangle f l g G$ or the $\triangle m o t A B$ mutant into $G$. mellonella larvae and incubated the larvae at $25^{\circ} \mathrm{C}$ for either 3 or 21 days (all insects were dead after 2 days). The insects were then homogenised and the proportion of motile to non-motile bacteria was determined using swim agar, as described. After 3 days in the insect the $\Delta$ flgG mutant was present at slightly higher levels than the WT but this trend was reversed after 21 days at which time the WT predominated in the insect cadaver (Fig. 5A). Similarly the WT had almost completely out-competed the $\triangle m o t A B$ mutant 

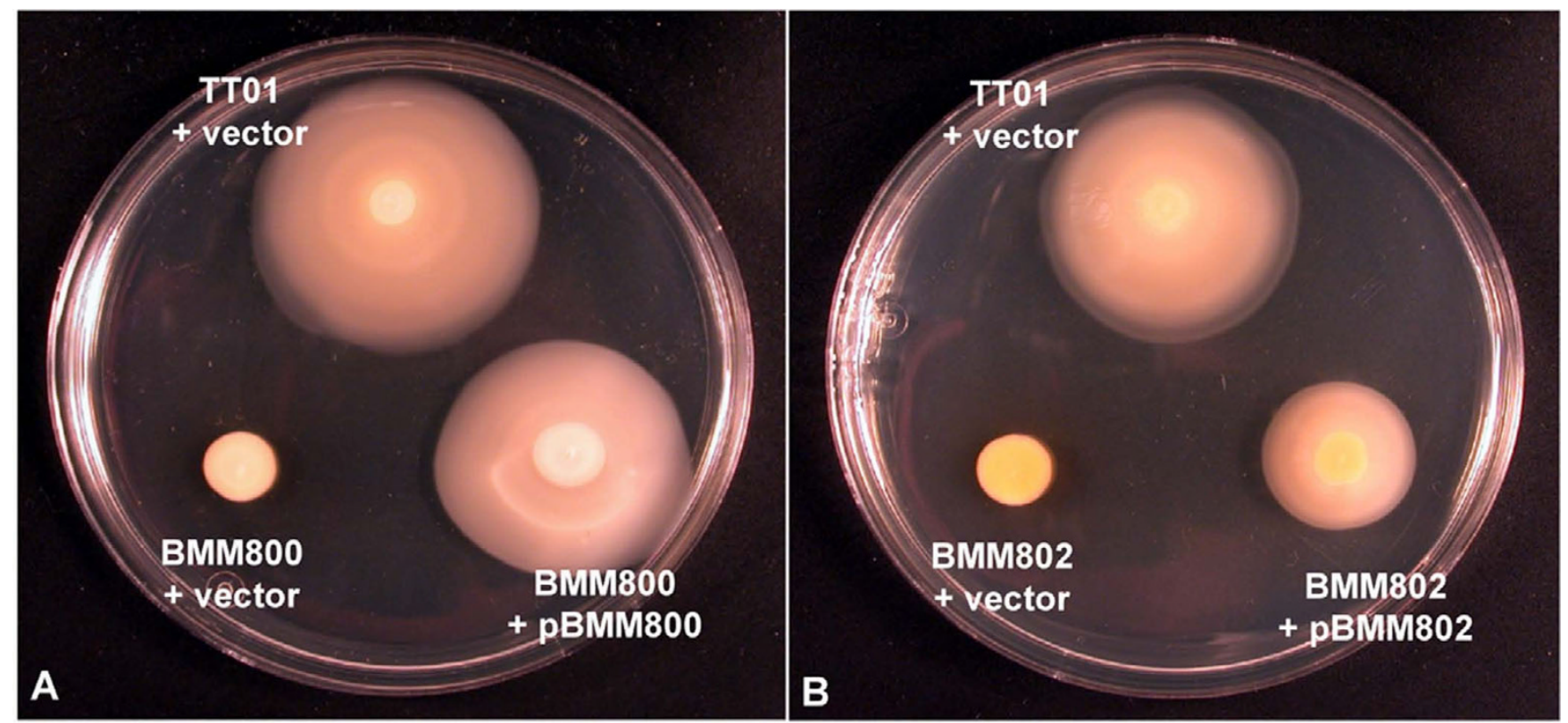

Figure 2

Swimming motility of $\boldsymbol{P}$. luminescens. The WT was transformed using the plasmid pTRC 99 a (TT0I + vector) and the mutants BMM800 $(\triangle \mathrm{flg} G)$ and BMM802 $(\triangle \operatorname{mot} A B)$ were transformed using $\mathrm{PTRC} 99 \mathrm{a}(\mathrm{BMM} 800+$ vector and $\mathrm{BMM802}+\mathrm{vector})$ or either pBMM800 (BMM800 + pBMM800) or pBMM802 (BMM802 + pBMM802). Cells were grown overnight at $30^{\circ} \mathrm{C}$ in $\mathrm{LB}$ broth (+ Amp) and diluted to an $O D_{600}=1.0$ in fresh LB before $5 \mu \mathrm{l}$ was spotted onto the surface of a swim agar plate. Plates were incubated at $30^{\circ} \mathrm{C}$ for $44 \mathrm{~h}$ before motility was scored.

after 21 days in the insect (Fig. 5B). Therefore motility confers a fitness advantage to Photorhabdus during the prolonged incubation in the insect normally experienced by these bacteria as part of their life-cycle. This would suggest that the cost associated with the production of flagella is offset by the ability of Photorhabdus to be motile in the insect.

\section{Motility affects the ability of Photorhabdus to colonise the nematode}

To test for a role for motility during the interaction with the nematode we inoculated lipid agar plates with overnight cultures of TT01, BMM800 ( $\triangle$ flgG) or BMM802 $(\triangle m o t A B)$. After 3 days at $25^{\circ} \mathrm{C}$ the bacterial biomass on the plates was seeded with 50 surface-sterilised IJ nematodes and incubated, in the dark, at $25^{\circ} \mathrm{C}$ for a total of 21 days. During this time 3 aspects of the bacteria-nematode interaction are routinely monitored: 1) the fraction of inoculated IJs that exit diapause to develop into adult hermaphrodites (i.e. this is called IJ recovery and it is an indicator of the ability of the bacteria to produce the signals required to stimulate IJ recovery); 2) the total number of new generation IJs that are recovered after 21 days (i.e. this is called the IJ yield and it is an indicator of the ability of the bacteria to support nematode growth and reproduction) and 3) IJ colonisation by the bacteria (required for the continuation of the mutualism between bacteria and nematode). We did not observe any defect in either IJ recovery or IJ yield suggesting that bacterial motility does not make a significant contribution to nematode growth and development (data not shown). On the other hand we did observe that the $\Delta f \lg G$ mutant was present in the IJ at levels significantly higher than the WT (median for $\Delta f l g G=159 \mathrm{cfu} / \mathrm{IJ}$ compared to median for WT $=105 \mathrm{cfu} /$ IJ; $P=0.0478$ ) whilst, in contrast, the $\triangle$ mot $A B$ mutant was present in the IJ at significantly lower levels than the WT (median for $\triangle m o t A B=82 \mathrm{cfu} / \mathrm{IJ}$ compared to median for $\mathrm{WT}=105 \mathrm{cfu} / \mathrm{IJ} ; P=0.0065$ ) (Fig. 6). Therefore, although not required for colonisation, the ability to produce flagella and motility do appear to have contrasting affects on the final number of bacteria present in the IJ.

\section{Discussion}

Photorhabdus are highly virulent to a wide range of insect larvae and, following insect death the bacteria must remain in the insect cadaver at high densities for extended periods of time (up to 2-3 weeks under optimal conditions) to facilitate nematode growth and development. We have shown that, although neither flagella production nor motility is required for pathogenicity, there is a significant advantage to being motile during the normally prolonged incubation in the insect. 


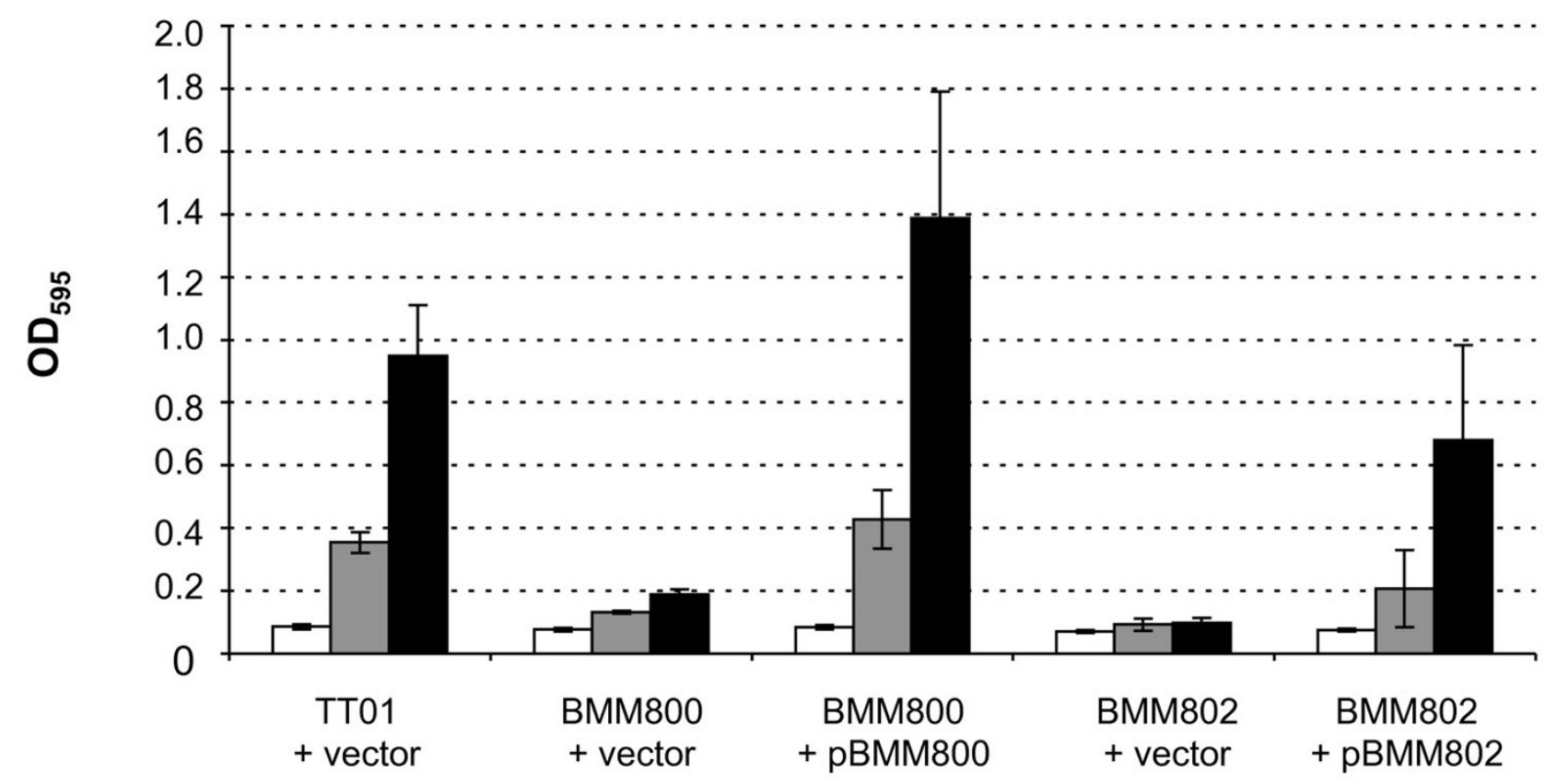

Figure 3

Biofilm formation by $\boldsymbol{P}$. luminescens. The cells (as indicated; see legend for Figure 2) were grown overnight at $30^{\circ} \mathrm{C}$ in $\mathrm{LB}$ broth (+ Amp). The $\mathrm{OD}_{600}$ of the culture was adjusted to 0.05 and $200 \mu \mathrm{l}$ was added to the well of a 96 -well Costar ${ }^{\circledR}$ PP microtitre plate. The plates were incubated for the appropriate time at $30^{\circ} \mathrm{C}$ before staining with crystal violet to quantify bacterial attachment. The results shown are the mean \pm SD of 3 experiments.

Our data show that, on agar plates, the $\triangle$ mot $A B$ mutant is as competitive as the WT suggesting that there is no perceived benefit to being motile in this environment. This was not unexpected as the concentration of agar used would prevent cells from swimming or swarming, whether they are motile or not, thus rendering the $\triangle$ mot $A B$ mutant neutral in this environment. On the other hand, the $\Delta f l g G$ mutant has a considerable advantage over WT suggesting that there is a metabolic cost associated with the production of flagella. In a recent study Fontaine et al (2008) showed that the reduced mortality associated with a fliA mutation in E. coli was probably due to decreased internal cell stress due to the absence of physical destabilisation of the membrane [21]. Therefore the cost associated with flagella production in Photorhabdus may be due to the utilisation of resources and energy for the production and/or function of the flagella or it may be due to the stresses associated with assembly of the flagella through the cellular membrane. In the insect this trend is reversed and the $\Delta f l g G$ cells are now disadvantaged during extended growth (i.e. 21 days). This would suggest that flagella production benefits the bacteria during insect infection. Therefore the costs of flagella production (and presumably motility) appear to be offset during extended incubation in the insect. In support of this, the $\triangle m o t A B$ mutant, that still bears the cost of producing flagella and yet does not derive any benefit associated with motility in the insect, is present at much lower levels than the $\Delta f \lg G$ mutant in competition assays after 21 days.

The frequency of motile cells observed throughout the growth of Photorhabdus under normal culturing conditions (i.e. shaking at $30^{\circ} \mathrm{C}$ in LB broth) is very low ( $<<$ $1 \%$ ) (our unpublished data). During growth under static conditions the frequency of motility in the population increased reaching a maximum of $30 \%$ at $16 \mathrm{~h}$ post-inoculation before rapidly decreasing (data not shown). Therefore motility is restricted, both spatially and temporally, during the growth of Photorhabdus. This limited exposure of motility to any selection pressure in the environment could explain the relatively modest differences in fitness observed in this study.

In uropathogenic E. coli (UPEC) motility has been shown to contribute to the fitness of the bacteria during colonisation of the urinary tract [22]. Indeed recent studies have shown that motility facilitates the movement of UPEC to the upper urinary tract [12]. Moreover motility has been shown to be required for Salmonella to access high-energy nutrients found at sites of inflammation in the mamma- 

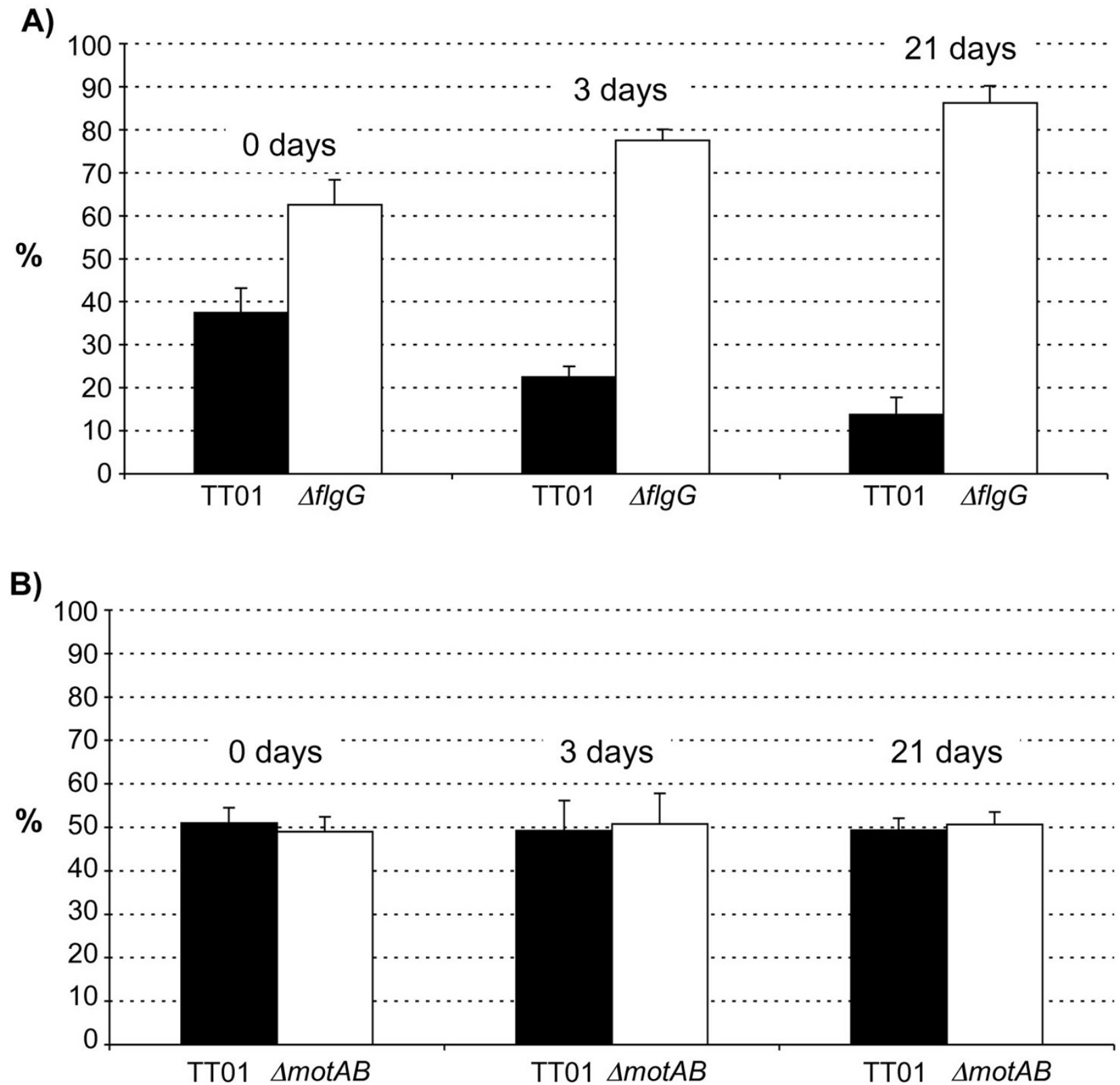

Figure 4

Competition assays on agar plates. Equal numbers of $\mathrm{A}) \mathrm{TTOI}$ and $\mathrm{BMM} 800(\Delta f \mathrm{gG})$ and $\mathrm{B})$ TT0I and $\mathrm{BMM} 802(\Delta m o t A B)$ were mixed and inoculated onto each of 5 lipid agar plates. The proportion (\%) of motile:non-motile bacteria was determined in the initial mixture before inoculation and this is reported as Day 0. At the other time points the proportion of motile:nonmotile bacteria was determined on each of the 5 individual plates. The results shown are the mean \pm SD of 3 experiments.

lian gut [13]. In the same way we expect that motility in Photorhabdus, a closely related bacterium, will facilitate the movement of the bacteria throughout the insect cadaver thus enabling invasion of nutrient-rich niches that facilitate growth of the motile strain. In this regard Photorhab$d u s$ would also be expected to undergo chemotaxis and the genome is predicted to encode a complete Che signal- ling system in addition to 2 methyl-accepting chemotaxis proteins i.e. MCPs (see Fig. 1). However the role of chemotaxis was not investigated in this study.

We have shown that neither flagella production nor motility is required for the mutualistic association between Photorhabdus and the Heterorhabditis nematode. 


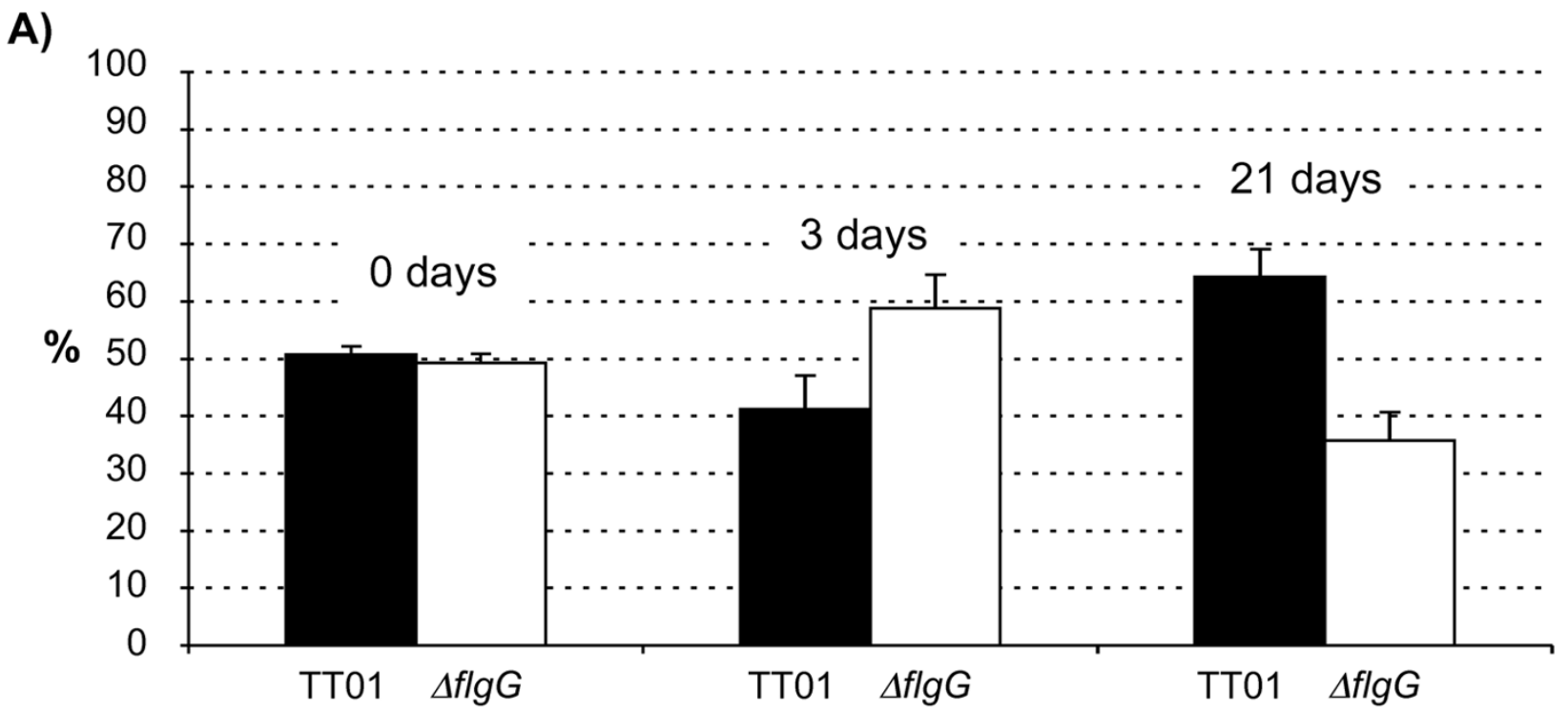

B)

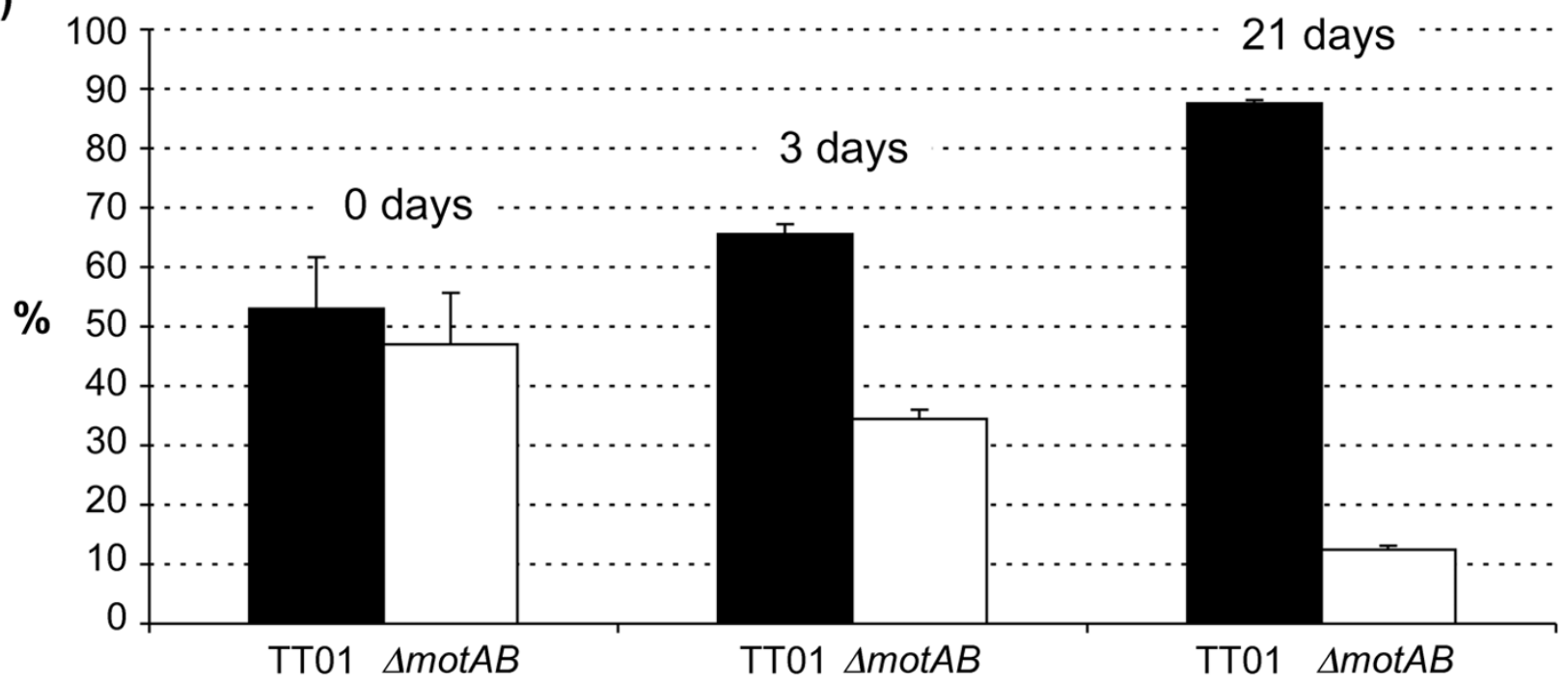

\section{Figure 5}

Competition assays in the insect. Equal numbers of A) TTOI and BMM800 ( $\triangle f l g G)$ and B) TTOI and BMM802 $(\Delta m o t A B)$ were mixed and injected into $G$. mellonella larvae. The proportion (\%) of motile:non-motile bacteria was determined in the initial mixture before injection and this is reported as Day 0. At Day 3 and Day 21 the proportion of motile:non-motile bacteria was determined in each of 10 individual insects. The results shown are the mean \pm SD of 3 experiments.

Photorhabdus has 2 roles during the interaction with the nematode: 1) the bacteria must provide nutrients for the nematode and 2) the bacteria must colonise the IJ. The nematodes feed on the Photorhabdus biomass that is present either in the insect or on agar plates and, therefore, the nematodes obtain a substantial part of their required nutrients directly from the bacteria. We did not see any differences in the growth and development of the nematodes growing on agar plates with the WT bacteria compared to the $\triangle$ flgG and $\triangle \operatorname{mot} A B$ mutants. Indeed infecting insects with IJs colonised with either $\triangle$ flgG or $\triangle m o t A B$ mutants resulted in an IJ yield (a quantitative indicator of the ability of the bacteria to support nematode growth and development) very similar to that observed with IJs colonised with WT bacteria (data not shown). Therefore flagella production and motility do not appear to play any role in the nutritional interaction 


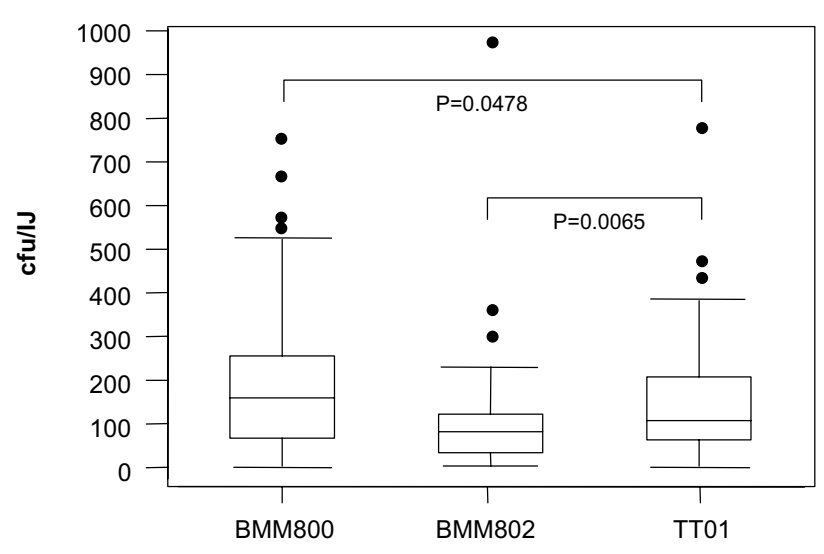

Figure 6

Bacterial levels in IJ stage nematodes. IJ nematodes were harvested from lipid agar plates inoculated with TTO I, BMM800 ( $\triangle$ flgG) or BMM802 ( $\triangle$ motAB). Individual IJs were crushed and the homogenate was plated on LB (Rif) plates to determine the bacterial population per IJ (cfu/lJ). Three independent overnight cultures for each strain were prepared and each overnight was inoculated onto 4 lipid agar plates. After 21 days incubation at $25^{\circ} \mathrm{C} 10 \mathrm{Jj}$ from each plate $(\mathrm{n}=$ 120 for each bacterial strain) were homogenised and the number of cfu/lJ was determined by plating the homogenate on LB agar (Rif). Any nematodes that were not colonised were not included in further analyses. Therefore, for IJs grown on WT bacteria $\mathrm{n}=100$, for IJs grown on the $\triangle$ flgG mutant $\mathrm{n}=102$ and for IJs grown on the $\triangle \operatorname{mot} A B$ mutant $\mathrm{n}=$ 98. The data from these nematodes was analysed and compared to WT using the non-parametric Mann-Whitney $U$ test and the appropriate $P$ values are shown. The data is presented using a Box and Whisker format that shows the median value (horizontal line), the upper and lower quartiles (boxed) and the range of the data (whiskers). The remaining "outliers" are shown as solid circles.

between Photorhabdus and the nematodes either on agar plates or in the insect.

Photorhabdus are maternally transmitted from the hermaphrodite stage nematode (i.e. the mother) to the developing IJ [23]. As the Heterorhabditis nematodes feed on Photorhabdus some viable bacteria enter the lumen of the gut of the hermaphrodite and attach to specific cells in the distal region of the gut (specifically the INT9 cells). The bacteria infect the neighbouring rectal glands cells and replicate within vacuoles. The rectal gland cells rupture, releasing Photorhabdus into the body cavity of the hermaphrodite where the bacteria encounter and colonise the developing IJs [23]. The IJ is initially colonised by 1-2 bacteria that subsequently replicate, resulting in a final population of approximately $100 \mathrm{cfu}$ of WT bacteria per IJ. The proportion of IJs colonised by the $\triangle$ flgG and $\triangle m o t A B$ mutants is the same as the WT (i.e. approx. $80 \%$ in all cases) suggesting that attachment to the IJ, and presumably infection of the hermaphrodite, is independent of flagella and/or motility. On the other hand, the final population level of Photorhabdus within the IJ is significantly altered in nematodes that are cultured on either the $\triangle f l g G$ or the $\triangle \operatorname{mot} A B$ mutant strain. Therefore IJs that have been grown on the $\Delta f \lg G$ mutant carry, on average, a bacterial population that is $50 \%$ greater than the population within IJs cultured on WT bacteria. In contrast the $\triangle$ mot $A B$ mutant does not reach population levels within the IJ that are equivalent to the WT suggesting that the production of non-functional flagella negatively influences growth in the nematode. These results might be explained in terms of the metabolic cost associated with the production of flagella. Therefore, in the absence of flagella production, the $\Delta$ flgG mutant may be able to put more of the limited resources available within the nematode into biomass production and division. On the other hand the $\triangle m o t A B$ mutant still bears the cost of producing flagella although these are non-functional. Interestingly the fact that the $\triangle m o t A B$ mutation is not neutral, in terms of IJ colonisation, suggests that Photorhabdus are likely to be motile at some point during the colonisation of the nematode. The bacteria initially colonise the proximal end of the IJ gut and one possibility is that motility may facilitate exploration of the distal regions of the gut thus allowing the bacteria to make better use of the limited resources available within the nematode.

\section{Conclusion}

In this study we show that there is a significant metabolic cost associated with the production of flagella (and motility) in Photorhabdus. Nonetheless Photorhabdus are motile suggesting that motility is an adaptive trait that is under powerful positive selection in the environments where Photorhabdus is normally found i.e. in the insect and nematode. In this study we show that, although motility is not required for either pathogenicity or mutualism, this trait is advantageous during the interactions between Photothabdus and both of its invertebrate hosts. Therefore, in addition to describing a role for motility in Photorhabdus, this work also highlights the functional overlap between pathogenicity and mutualism and reinforces the utility of Photorhabdus as a model for studying these different bacteria-host interactions.

\section{Methods}

\section{Bacterial strains and culture conditions}

A spontaneous rifampicin-resistant mutant of Photorhabdus luminescens subsp. luminescens was used as the wildtype (WT) in all experiments [2]. The bacteria were cultured in LB broth or on LB agar (LB broth plus 1.5\% (w/ v) agar) at $30^{\circ} \mathrm{C}$ for $P$. luminescens. Unless otherwise stated all LB agar plates were supplemented with $0.1 \%(\mathrm{w} / \mathrm{v})$

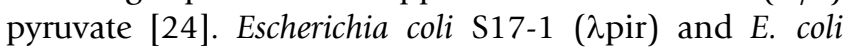


EC100 (Epicentre) were cultured at either $30^{\circ} \mathrm{C}$ or $37^{\circ} \mathrm{C}$ as indicated. Swim agar is LB broth plus $0.3 \%(\mathrm{w} / \mathrm{v})$ added agar. When required antibiotics were added at the following concentrations: ampicillin (Ap), $100 \mu \mathrm{g} \mathrm{ml}^{-1}$; chloramphenicol (Cm), $20 \mu \mathrm{g} \mathrm{ml}^{-1}$ and rifampicin (Rif), $50 \mu \mathrm{g}$ $\mathrm{ml}^{-1}$.

\section{Construction of deletion mutants}

The flgG and mot $A B$ genes were deleted using a previously described procedure [25]. This method results in the construction of unmarked and non-polar deletion mutation of the selected gene(s). For the deletion of each gene fragment A (approx. 600 bp upstream of the target gene) and fragment B (approx. $600 \mathrm{bp}$ downstream of the target gene) were amplified by PCR using KOD Hi-Fi polymerase (Novagen) and the primer pairs $\mathrm{KO} 1+\mathrm{KO} 3$ (for fragment A) and $\mathrm{KO} 2+\mathrm{KO} 4$ (for fragment $\mathrm{B}$ ). The primer sequences are listed in Table 1 . The fragments were purified using the Qiagen PCR clean up kit, combined and subjected to 10 cycles of primerless PCR to allow the polymerase to anneal fragments $\mathrm{A}$ and $\mathrm{B}$ (as $\mathrm{KO} 3$ and KO4 are complimentary). The annealed fragment was used as a template in a final PCR, using the appropriate $\mathrm{KO} 1$ and $\mathrm{KO} 2$ primers, to enrich for a full-length fragment. The different $\mathrm{KO} 1$ and $\mathrm{KO} 2$ primers incorporate restriction sites to facilitate cloning into the suicide vector pDS132 [26]. The resulting pDS132-flgG and pDS132motAB plasmids were isolated and used to transform $E$. coli S17-1 $\lambda$ pir (the donor) for conjugation into $P$. luminescens TT01 (the recipient). Conjugation and selection of the appropriate exconjugants was carried out as described previously [25]. Deletion mutants were then identified by subjecting selected exconjugants to colony PCR using the KO5 and KO6 primers (a deletion will result in a PCR product of a predictable size). The amplification product of each mutant was sequenced to confirm the integrity of the knock out allele.

\section{Cloning of the flgG and motAB genes}

The flgG and motAB genes were amplified from $P$. luminescens TT01 genomic DNA by PCR using KOD polymerase. The primers used for flgG were CAT001 (5'-TAAAACCCATGGTCCGATCATTATGGATTGC-3') and CAT002 (5'-GCTGGATCTAGATTATAACTGAGTCAGTTTTTGTAGC-3') and for motAB CAT003 (5'-GATATCCCATGGTAGTACTTTTAGGATATATC-3') and CAT004 (5'GCAGTGTCTAGATTACTTTGTCACCTTGGTCGG-3'). The flgG and motAB PCR fragments were digested with NcoI and XbaI and cloned into pTRC99a (Amersham Pharmacia Biotech) resulting in pBMM800 and pBMM802, respectively. The integrity and accuracy of all plasmid clones was verified by DNA sequencing.

\section{Biofilm formation}

The capacity of $P$. luminescens to form biofilms was assessed by measuring bacterial attachment to a plastic surface [2]. Strains were grown overnight in LB broth, diluted to $\mathrm{OD}_{600}=0.05$ in fresh $\mathrm{LB}$ and $200 \mu \mathrm{l}$ of the cell suspension was aliquoted in triplicate, into the wells of a Costar ${ }^{\circledast}$ polypropylene (PP) 96-well microtitre plate. The plates were sealed with a gas permeable membrane and incubated, without shaking in a saturated environment to prevent evaporation, at $30^{\circ} \mathrm{C}$. At the appropriate time the planktonic cells were removed by aspiration and the wells were washed with $1 \times$ phosphate buffered saline (PBS). To observe biofilm formation $250 \mu \mathrm{l}$ of $0.1 \%$ (w/v) crystal violet $(\mathrm{CV})$ was added to each well and the plates were incubated at room temperature for $20 \mathrm{~min}$ before rinsing 3 times with $1 \times$ PBS. To quantify biofilm formation the $\mathrm{CV}$ was dissolved in $250 \mu \mathrm{l}$ of $95 \%$ ethanol and the CV concentration was determined by measuring the $\mathrm{OD}_{595}$ using a Genios (Tecan) plate reader.

\section{Pathogenicity assays}

The pathogenicity of $P$. luminescens was assessed using Galleria mellonella larvae (the Greater Wax Moth) as the

Table I: Primers used for the construction of the $\triangle$ flgG and $\triangle$ mot $A B$ mutants

\begin{tabular}{ll}
\hline Primer & Sequence $\left(5^{\prime}-3^{\prime}\right)^{\text {a }}$ \\
\hline KOIflgG & ATTATGCATGCCGATGAACTATACCTCTCGTCCG \\
KO2flgG & ATATAGAGCTCGCCAGTAACCGATCGACTGTCACG \\
KO3flgG & GACGATTAGGTTACATCGGTTTTATCCTCTGTGTC \\
KO4flgG & GGATAAAACCGATGTAACCTAATCGTCAATCAG \\
KO5flgG & GCAACTGGCTGCTTTGCGAGC \\
KO6flgG & GTCCGTGGGTTAACGACACCG \\
KOImotAB & TATGCATGCGTTACAAATGCTTGAAAGTGAAACAC \\
KO2motAB & ATATAGAGCTCGTGGCTGATGTTACCAATGATGC \\
KO3motAB & CGTTACTTTGTCACCTTGGTCGCACGCGATATCCTTTAGC \\
KO4motAB & GCTAAAGGATATCGCGTGCGACCAAGGTGACAAAGTAACG \\
KO5motAB & GCAGCTTACTAGGGAATCTCGAGTGG \\
KO6motAB & CTGCCCGTTGACGTGGCGATCCCG \\
\hline
\end{tabular}

a restriction sites are underlined 
model insect host. The G. mellonella larvae were purchased from Livefood (UK). Briefly overnight cultures of $P$. luminescens TT01 were washed 3 times in $1 \times$ PBS before the $\mathrm{OD}_{600}$ was adjusted to 1.0 as this has been shown to be equivalent to $4 \times 10^{8} \mathrm{cfu} \mathrm{ml}^{-1}$ (our unpublished data). The culture was diluted with $1 \times$ PBS to give cell density of $2 \times$ $10^{4} \mathrm{cfu} \mathrm{ml}^{-1}$ and $10 \mu \mathrm{l}$ (equivalent to $200 \mathrm{cfu}$ ) was injected into the hemolymph of a G. mellonella larva using a Hamilton syringe and a BD Microlance ${ }^{\mathrm{TM}} 330 \mathrm{G} \times 1 / 2^{\text {" needle. }}$ For competition assays the WT and mutant strain were grown overnight in $\mathrm{LB}$ at $30^{\circ} \mathrm{C}$ and equal numbers of cells from each culture were mixed and subsequently diluted so that 200 cfu could be injected into each insect larva. The proportion of WT and mutant bacteria in the injection mixture was assessed by plating an aliquot of the mixture onto LB (Rif) agar followed by patching 100 colonies onto swim agar. To determine the proportion of WT and mutant bacteria in the insect an infected insect larvae was surface sterilised by dipping in ethanol and passing the insect through a Bunsen flame before quickly plunging it into $5 \mathrm{ml}$ sterile $1 \times$ PBS in a universal tube. The insect cadaver was sliced open using a sterile scalpel blade and homogenised by adding 7 (3-4 $\mathrm{mm})$ sterile glass beads to the tube, followed by vortexing for $2 \mathrm{~min}$. The supernatant, containing the bacteria, was plated onto LB (Rif) agar and the proportion of motile/non-motile bacteria was determined by patching 50 colonies onto swim agar.

\section{In vitro symbiosis assays}

An aliquot of $50 \mu \mathrm{l}$ of an overnight culture diluted to an $\mathrm{OD}_{600}=1.0$ of the appropriate bacteria was spread, in $\mathrm{a} Z$ pattern, onto the surface of a lipid agar plate using an inoculating loop. The plates were incubated at $30^{\circ} \mathrm{C}$ for 3 days before adding 50 surface sterilised IJ nematodes to the bacterial biomass. Nematodes were surface-sterilised by washing in a solution $(0.4 \%(\mathrm{w} / \mathrm{v}))$ of hyamine (Sigma). Nematode recovery was assessed 7 days after addition of IJs by counting the number of hermaphrodites on the lipid agar plate. The new generation of IJs migrate to the lid of the Petri dish and, after 21 days, these nematodes were collected, by washing the lid with PBS to a final volume of $50 \mathrm{ml}$, and the number of IJs present (i.e the IJ yield) was determined. In competition assays the assays were the same except that the lipid agar plates were inoculated with equal numbers of the WT and mutant bacteria. At 3 and 21 days post-inoculation the bacterial biomass was scraped off the plate and the proportion of motile/non-motile bacteria was determined (as before). Colonisation levels in the IJ were determined by crushing single, surface-sterilised IJ nematodes in $100 \mu \mathrm{l} 1 \times$ PBS using a hand-held homogeniser and plating the homogenate onto LB (Rif) agar.

\section{Authors' contributions}

CAE undertook all of the experiments described in this manuscript. DJC conceived of the study, designed the experiments and drafted the manuscript. All authors read and approved the final manuscript.

\section{Acknowledgements}

The work outlined in this study was carried out equally in the University of Bath and University College Cork. The research was funded through the Exploiting Genomics initiative of the BBSRC in the UK (86/EGAI6I83) and Science Foundation Ireland. CAE is supported by a PhD fellowship from the University of Bath. The authors would also like to thank Susan Joyce for technical help and advice during this study.

\section{References}

I. Brugirard-Ricaud K, Duchaud E, Givaudan A, Girard PA, Kunst F, Boemare N, Brehelin M, Zumbihl R: Site-specific antiphagocytic function of the Photorhabdus luminescens type III secretion system during insect colonization. Cell Microbiol 2005, 7:363-37I.

2. Bennett HPJ, Clarke DJ: The pbgPE operon in Photorhabdus luminescens is required for pathogenicity and symbiosis. J Bacteriol 2005, I 87:77-84.

3. Kim Y, Ji D, Cho S, Park Y: Two groups of entomopathogenic bacteria, Photorhabdus and Xenorhabdus, share an inhibitory action against phospholipase $A 2$ to induce host immunodepression. J Invertebr Pathol 2005, 89:258-264.

4. Waterfield NR, Bowen DJ, Fetherston JD, Perry RD, ffrench-Constant RH: The tc genes of Photorhabdus: a growing family. Trends Microbiol 2001, 9:185-191.

5. Daborn PJ, Waterfield N, Blight MA, Ffrench-Constant RH: Measuring virulence factor expression by the pathogenic bacterium Photorhabdus luminescens in culture and during insect infection. J Bacteriol 200I, I 83:5834-5839.

6. ffrench-Constant R, Waterfield N, Daborn P, Joyce S, Bennett $H, A u$ C, Dowling A, Boundy S, Reynolds S, Clarke D: Photorhabdus: towards a functional genomic analysis of a symbiont and pathogen. FEMS Microbiol Rev 2003, 26:433-456.

7. Goodrich-Blair H, Clarke DJ: Mutualism and pathogenesis in Xenorhabdus and Photorhabdus: two roads to the same destination. Mol Microbiol 2007, 64:260-268.

8. Aldridge P, Hughes KT: Regulation of flagellar assembly. Curr Opin Microbiol 2002, 5: 160-165.

9. Chevance FF, Hughes KT: Coordinating assembly of a bacterial macromolecular machine. Nat Rev Microbiol 2008, 6:455-465.

10. Millikan DS, Ruby EG: Alterations in Vibrio fischeri motility correlate with a delay in symbiosis initiation and are associated with additional symbiotic colonization defects. Appl Environ Microbiol 2002, 68:2519-2528.

II. Gauger EJ, Leatham MP, Mercado-Lubo R, Laux DC, Conway T, Cohen PS: Role of motility and the flhDC operon in Escherichia coli MG I 655 colonization of the mouse intestine. Infect Immun 2007, 75:3315-3324.

12. Lane MC, Alteri CJ, Smith SN, Mobley HLT: Expression of flagella is coincident with uropathogenic Escherichia coli ascension to the upper urinary tract. Proc Natl Acad Sci (USA) 2007, I04:16669-16674.

13. Stecher B, Barthel M, Schlumberger MC, Haberli L, Rabsch W, Kremer M, Hardt W-D: Motility allows S. Typhimurium to benefit from the mucosal defence. Cell Microbiol 2008, I 0: I I 66- I I80.

14. Millikan DS, Ruby EG: Vibrio fischeri flagellin A is essential for normal motility and for symbiotic competence during initial squid light organ colonization. J Bacteriol 2004, I 86:43 I5-4325.

15. Hall AR, Colegrave N: Decay of unused characters by selection and drift. J Evol Biol 2008, 21:610-617.

16. Duchaud E, Rusniok C, Frangeul L, Buchrieser C, Givaudan A, Taourit S, Bocs S, Boursaux-Eude C, Chandler M, Charles JF, et al.: The genome sequence of the entomopathogenic bacterium Photorhabdus luminescens. Nat Biotechnol 2003, 2 I : | 307- | 3 | 3.

17. O'Toole GA, Kolter R: Flagellar and twitching motility are necessary for Pseudomonas aeruginosa biofilm development. Mol Microbiol 1998, 30:295-304. 
18. Pratt LA, Kolter R: Genetic analysis of Escherichia coli biofilm formation: roles of flagella, motility, chemotaxis and type I pili. Mol Microbiol 1998, 30:285-293.

19. Harshey RM: Bacterial motility on a surface: many ways to a common goal. Annu Rev Microbiol 2003, 57:249-273.

20. Wood TK, Gonzalez Barrios AF, Herzberg M, Lee J: Motility influences biofilm architecture in Escherichia coli. Appl Microbiol Biotechnol 2006, 72:36I-367.

21. Fontaine F, Stewart EJ, Lindner AB, Taddei F: Mutations in two global regulators lower individual mortality in Escherichia coli. Mol Microbiol 2008, 67:2-14.

22. Lane MC, Lockatell V, Monterosso G, Lamphier D, Weinert J, Hebel $\mathrm{JR}$, Johnson DE, Mobley HLT: Role of motility in the colonization of uropathogenic Escherichia coli in the urinary tract. Infect Immun 2005, 73:7644-7656.

23. Ciche TA, Kim K, Kaufmann-Daszczuk B, Nguyen KCQ, Hall DH: Cell invasion and matricide during Photorhabdus luminescens transmission by Heterorhabditis bacteriophora nematodes. Appl Environ Microbiol 2008, 74:2275-2287.

24. Xu J, Hurlbert RE: Toxicity of irradiated media for Xenorhabdus spp. Appl Environ Microbiol 1990, 56:815-818.

25. Brachmann AO, Joyce SA, Jenke-Kodoma H, Schwar G, Clarke DJ, Bode HB: A type II polyketide synthase is responsible for anthraquinone biosynthesis in Photorhabdus luminescens. Chembiochem 2007, 8:172I-1728.

26. Philippe N, Alcaraz J-P, Coursange E, Geiselmann J, Schneider D: Improvement of pCVD442, a suicide plasmid for gene allele exchange in bacteria. Plasmid 2004, 5 I:246-255.

Publish with Biomed Central and every scientist can read your work free of charge

"BioMed Central will be the most significant development for disseminating the results of biomedical research in our lifetime. "

Sir Paul Nurse, Cancer Research UK

Your research papers will be:

- available free of charge to the entire biomedical community

- peer reviewed and published immediately upon acceptance

- cited in PubMed and archived on PubMed Central

- yours - you keep the copyright

Submit your manuscript here:

http://www.biomedcentral.com/info/publishing_adv.asp
BioMedcentral 